\title{
Online Optimal Power Flow With Renewables
}

\author{
Seung-Jun Kim \\ Dept. of CSEE \\ University of Maryland, Baltimore County \\ Baltimore, MD 21250, USA \\ sjkim@umbc.edu
}

\author{
Geogios B. Giannakis \\ Dept. of ECE \\ University of Minnesota \\ Minneapolis, MN 55455, USA \\ georgioseumn.edu
}

\author{
Kwang Y. Lee \\ Dept. of ECE \\ Baylor University \\ Waco, TX 76798, USA \\ Kwang_Y_Lee@baylor.edu
}

\begin{abstract}
Optimal power flow (OPF) is a critical control task for reliable and efficient operation of power grids. Significant challenges are anticipated in the development of future power systems, as a substantial amount of inherently uncertain renewable resources are incorporated, imposing volatile dynamics to the grid. In this work, an online learning approach, which does not require elaborate models for uncertainty, yet is capable of providing a provable performance guarantee, is adopted to tackle the OPF with renewables in an online fashion. A twostage procedure is considered, where the conventional generation level is committed before the renewable output is revealed, followed by spot market transactions to account for imbalance. Simulated tests with a 30-bus case show that, under high variability of renewables, the proposed hedging scheme beats a static alternative, which solves two OPF problems per time slot.
\end{abstract}

\section{INTRODUCTION}

Optimal power flow (OPF) is a critical control task for efficient and reliable operation of power systems [1], [2]. The goal of OPF is to determine economical operating parameters for power systems, minimizing various costs, such as the generation cost, line losses, and even consumer disutility, subject to algebraic network constraints capturing the relationship between the power variables and the voltage variables. At the same time, the physical operational constraints at buses and lines, as well as the power balancing constraints between generated and comsumed power, must be satisfied.

Due to the quadratic relation between the power-related quantities and the voltage phasors, AC OPF constitutes a challenging nonconvex problem. Thus, various nonlinear programming and heuristic optimization approaches have been developed [3], [4], [5]. (Non)linear programming approaches such as the ones based on gradient descent, Newton-Raphson, linear programming, and interior-point methods, essentially seek points satisfying the Karush-Kuhn-Tucker (KKT) condition for local optimality. Thus, the quality of these solutions is often sensitive to initialization [2]. Heuristic alternatives, such as the genetic algorithm, evolutionary programming, and particle swarm optimization, can potentially achieve globally optimal solutions, but at significantly higher computational cost [5].

Work in this paper was supported in part by NSF grants 1343248,1423316 and 1442686, and by the Institute of Renewable Energy and the Environment (IREE) at the University of Minnesota, under grant No. RL-0010-13.
Convex relaxation approaches for OPF have attracted much attention recently as a viable technique to obtain globally optimal solutions with reasonable computational complexity [6], [7], [8], [9]. In particular, a semidefinite programming (SDP) relaxation of the OPF problem with a quadratic generation cost is dual-to-dual of the original nonconvex OPF problem. It has been shown that for many setups of practical interest, the SDP relaxation possesses zero duality gap, although there exist practical exceptions [10]. Implementation of the SDPbased OPF for large-scale power systems was studied in [11].

Incorporation of renewable energy resources is becoming an indispensible element of power system operation. Although clean and economically attractive, the renewable energy resources are not dispatchable at will. They are also difficult and costly to forecast accurately. Therefore, it is imperative to build robustness to power system operation against the uncertainties of the renewables. Stochastic and robust optimization techniques have been employed to cope with this uncertainty and minimize operational costs [12], [13]. However, such approaches typically require characterizing the uncertainty bounds or the distribution of the renewable generation output.

An alternative is to take an online and adaptive approach of adjusting the operational parameters in real time. Dynamic stochastic OPF with an actor/critic model was proposed in [14]. Model predictive control approaches were advocated in [15] and [16] under high penetration of renewables. OPF with real-time closed-loop control was proposed in [17]. A Markov decision process framework was adopted in [18]. However, these approaches either involve elaborate models for system dynamics, or they incur significant computational cost to take into account future system states. Thus, they may be feasible only for small-scale systems, or for large-scale systems with considerable sacrifice of optimality.

The online convex optimization (OCO) framework is widely used in the machine learning community due to its strong performance guarantees even without elaborate models on the dynamics [19]. As the performance guarantees do not require stochastic models or detailed assumptions on disturbances, the algorithms are applicable even to adversarial setups, where the disturbances are chosen strategically to beat the purpose of the learner. This aspect highlights the robustness of the approach. The OCO framework was adopted for economic dispatch (ED) of microgrids in [20], where it was shown that online ED was effective against the high volatility of wind 
turbine generation. The approach was shown to be useful for power system state estimation (PSSE) as well as for demand response setups in [21], [22].

The contribution of this work is to develop an OCO approach for OPF with renewable generation. The setup for online OPF involves discrepancy of the time when the decision is made and the time when the necessary information is revealed. That is, by the beginning of a time slot, the output levels of conventional generators must be determined (and fixed) without knowledge of actual realization of various uncertain quantities such as renewable energy production, loads, and spot market prices. At the end of the time slot, these quantities are revealed so that the additional cost (or profit if one could sell surplus energy to the spot market) due to the purchase of deficit power from the spot market can be assessed. Such a model reflects precisely the setup for OCO. To properly accommodate the nonconvexity of AC OPF in the OCO framework, a convex relaxation technique for OPF is employed. The simulated tests show that the performance of the online OPF, which solves a single OPF problem per time slot, is superior to that of a two-stage static OPF, which solves two OPF problems per slot, when the renewable outputs fluctuate significantly.

The rest of the paper is organized as follows. In Section II, the static OPF formulation and its convex relaxation are introduced. Online OPF formulation and its solution method are provided in Section III. The results of simulated tests are discussed in Section IV. Conclusions and future research directions are offered in Section V.

\section{STATIC OPF}

\section{A. OPF Formulation}

The aim of OPF is to optimize certain economic objectives such as the generation cost and line losses, subject to various bus and line operational constraints, while meeting the demand [2]. Let $\mathcal{N}:=\{1,2, \ldots, N\}$ denote the set of buses (nodes) in a power grid consisting of $N$ buses, and $\mathcal{E}$ the lines (edges) between the buses. Let $\mathcal{N}_{n}:=\left\{n^{\prime}\right.$ : $\left.\left(n, n^{\prime}\right) \in \mathcal{E}\right\}$ be the set of neighboring buses of $n$. Denote the voltages $\left\{V_{n}\right\}_{n \in \mathcal{N}}$ at all buses by $\mathbf{v}:=\left[V_{1}, V_{2}, \ldots, V_{N}\right]^{T}$, and the currents injected into individual buses $\left\{I_{n}\right\}_{n \in \mathcal{N}}$ by $\mathbf{i}:=\left[I_{1}, I_{2}, \ldots, I_{N}\right]^{T}$, where ${ }^{T}$ stands for transposition. For each line $\left(n, n^{\prime}\right) \in \mathcal{E}$, the admittance is denoted as $y_{n n^{\prime}}$, and the admittance matrix $\mathbf{Y} \in \mathbb{C}^{N \times N}$ is formed with $\left(n, n^{\prime}\right)$ entry

$$
Y_{n n^{\prime}}= \begin{cases}\sum_{n^{\prime} \in \mathcal{N}_{n}} y_{n n^{\prime}}, & \text { if } n=n^{\prime} \\ -y_{n n^{\prime}}, & \text { if } n \neq n^{\prime}, \quad\left(n, n^{\prime}\right) \in \mathcal{E} \\ 0, & \text { otherwise. }\end{cases}
$$

Then, Kirchhoff's and Ohm's laws yield

$$
I_{n}=\sum_{n^{\prime} \in \mathcal{N}_{n}}\left(V_{n}-V_{n^{\prime}}\right) y_{n n^{\prime}}
$$

which can be written compactly as $\mathbf{i}=\mathbf{Y v}$. The complex power injected to node $n \in \mathcal{N}$ is given by

$$
S_{n}:=P_{n}+j Q_{n}=V_{n} I_{n}^{*}=\sum_{n^{\prime} \in \mathcal{N}_{n}} V_{n}\left(V_{n}-V_{n^{\prime}}\right)^{*} y_{n n^{\prime}}^{*}
$$

where $j:=\sqrt{-1}, *$ denotes complex conjugation, and $P_{n}$ $\left(Q_{n}\right)$ represents the real (reactive) power injected to bus $n$.

Let $\mathcal{N}_{g}$ be the set of buses with conventional generators, and $P_{g, n}$ and $Q_{g, n}$ the deterministic active and the reactive powers, respectively, generated at bus $n \in \mathcal{N}_{g}$. If $n \notin \mathcal{N}_{g}$, then $P_{g, n}=Q_{g, n}=0$ by convention. Likewise, $P_{l, n}$ and $Q_{l, n}$ denote the active and the reactive powers, respectively, consumed by the load at bus $n$, with $P_{l, n}=Q_{l, n}=0$ if bus $n$ does not have any load. Finally, let $P_{r, n}$ and $Q_{r, n}$ be the inherently random active and the reactive powers, respectively, produced by renewable generation at buses $n \in \mathcal{N}_{r}$. Then, the power balance condition can be written as

$$
\begin{gathered}
P_{n}=P_{g, n}-P_{l, n}+P_{r, n}, \quad n \in \mathcal{N} \\
Q_{n}=Q_{g, n}-Q_{l, n}+Q_{r, n}, \quad n \in \mathcal{N} .
\end{gathered}
$$

The OPF problem can be formulated as minimizing a certain cost incurred at each bus, while meeting the power flow constraints (3) and the balacing conditions (4), as well as various operational constraints. Specifically, OPF aims at

$$
\min _{\mathbf{v},\left\{P_{g, n}\right\},\left\{Q_{g, n}\right\}} \sum_{n \in \mathcal{N}} f_{n}\left(P_{g, n}\right)
$$

subject to (3), (4), and

$$
\begin{aligned}
& \underline{V}_{n} \leq\left|V_{n}\right| \leq \bar{V}_{n}, \quad n \in \mathcal{N} \\
& \underline{P}_{g, n} \leq P_{g, n} \leq \bar{P}_{g, n}, \quad n \in \mathcal{N}_{g} \\
& \underline{Q}_{g, n} \leq Q_{g, n} \leq \bar{Q}_{g, n}, \quad n \in \mathcal{N}_{g} \\
& \left|V_{n}-V_{n^{\prime}}\right| \leq \bar{V}_{n n^{\prime}}, \quad\left(n, n^{\prime}\right) \in \mathcal{E} .
\end{aligned}
$$

Here, $\underline{V}_{n}$ and $\bar{V}_{n}$ are the lower and upper limits, respectively, on the voltage magnitude at bus $n$, and (5c) and (5d) specify the acceptable ranges for the real and the reactive power generation, respectively, at buses $n \in \mathcal{N}_{g}$. Eq. (5e) captures the line capacity, or the maximum phase difference over the lines. The objective in (5a) can capture various costs such as the generation cost, line losses, conservation voltage reduction (CVR)-related cost, and even consumer disutility. For example, the generation cost is often modeled by quadratic functions as

$$
f_{n}\left(P_{g, n}\right):=c_{2, n} P_{g, n}^{2}+c_{1, n} P_{g, n}+c_{0, n}, \quad n \in \mathcal{N}_{g}
$$

where $c_{2, n} \geq 0$. Problem (5) is nonconvex due to the quadratic relation between the power variables and the voltage variables as manifested in (3).

\section{B. Convex Relaxation Approach to OPF}

An approach that has received renewed attention recently is to solve convex relaxations of (5) using SDP and second-order cone programming (SOCP) relaxations [6], [8], [9]. The SDP relaxation is obtained by noticing that the relevant quantities in (5) can be represented as linear in a matrix variable $\mathbf{X}:=$ 
$\mathbf{v} \mathbf{v}^{H}$ [7]. To see this, let $\mathbf{e}_{n}$ denote the $n$-th canonical vector in $\mathbb{R}^{N}$. Then, it can be verified that

$$
\begin{aligned}
S_{n} & =\mathbf{e}_{n}^{T} \mathbf{v}\left(\mathbf{e}_{n}^{T} \mathbf{Y} \mathbf{v}\right)^{H}=\operatorname{tr}\left\{\mathbf{X Y} \mathbf{Y}^{H} \mathbf{e}_{n} \mathbf{e}_{n}^{T}\right\} \\
\left|V_{n}\right|^{2} & =X_{n n} \\
\left|V_{n}-V_{n^{\prime}}\right|^{2} & =X_{n n}+X_{n^{\prime} n^{\prime}}-X_{n n^{\prime}}-X_{n^{\prime} n}
\end{aligned}
$$

where $X_{n n^{\prime}}$ is the $\left(n, n^{\prime}\right)$-entry of $\mathbf{X}$. Thus, upon defining

$$
\begin{aligned}
\mathbf{Y}_{n} & :=\mathbf{e}_{n} \mathbf{e}_{n}^{T} \mathbf{Y} \\
\overline{\mathbf{Y}}_{n} & :=\frac{1}{2}\left(\mathbf{Y}_{n}+\mathbf{Y}_{n}^{H}\right) \\
\tilde{\mathbf{Y}}_{n} & :=\frac{j}{2}\left(\mathbf{Y}_{n}-\mathbf{Y}_{n}^{H}\right)
\end{aligned}
$$

one can identify

$$
\begin{aligned}
P_{n} & =\operatorname{tr}\left\{\mathbf{X} \overline{\mathbf{Y}}_{n}\right\} \\
Q_{n} & =\operatorname{tr}\left\{\mathbf{X} \tilde{\mathbf{Y}}_{n}\right\} .
\end{aligned}
$$

Therefore, (5) can be rewritten equivalently as

$$
\min _{\mathbf{X} \succeq 0} \sum_{n \in \mathcal{N}_{g}} f_{n}\left(\operatorname{tr}\left\{\mathbf{X} \overline{\mathbf{Y}}_{n}\right\}+P_{l, n}-P_{r, n}\right)
$$

subject to:

$$
\begin{aligned}
& \underline{V}_{n}^{2} \leq X_{n n} \leq \bar{V}_{n}^{2}, \quad n \in \mathcal{N} \\
& \underline{P}_{n} \leq \operatorname{tr}\left\{\mathbf{X} \overline{\mathbf{Y}}_{n}\right\} \leq \bar{P}_{n}, \quad n \in \mathcal{N} \\
& \underline{Q}_{n} \leq \operatorname{tr}\left\{\mathbf{X} \tilde{\mathbf{Y}}_{n}\right\} \leq \bar{Q}_{n}, \quad n \in \mathcal{N} \\
& X_{n n}+X_{n^{\prime} n^{\prime}}-X_{n n^{\prime}}-X_{n^{\prime} n} \leq \bar{V}_{n n^{\prime}}^{2}, \quad\left(n, n^{\prime}\right) \in \mathcal{E} \\
& \operatorname{rank}\{\mathbf{X}\}=1
\end{aligned}
$$

where $\underline{P}_{n}:=\underline{P}_{g, n}-P_{l, n}+P_{r, n}$ and $\bar{P}_{n}:=\bar{P}_{g, n}-P_{l, n}+P_{r, n}$ (and likewise for $Q_{n}$ and $\bar{Q}_{n}$ ) for $n \in \mathcal{N}_{g}$, while for $n \notin \mathcal{N}_{g}$, $\bar{P}_{n}=\underline{P}_{n}:=P_{l, n}-P_{r, n}$ and $\bar{Q}_{n}=\underline{Q}_{n}:=Q_{l, n}-Q_{r, n}$.

This problem would be convex if it were not for (12f). Neglecting this nonconvex constraint yields a convex relaxation. In fact, upon defining $\tilde{c}_{2, n}:=c_{2, n}, \tilde{c}_{1, n}:=2 c_{2, n}\left(P_{l, n}-\right.$ $\left.P_{r, n}\right)+c_{1, n}$, and $\tilde{c}_{0, n}:=c_{2, n}\left(P_{l, n}-P_{r, n}\right)^{2}+c_{1, n}\left(P_{l, n}-\right.$ $\left.P_{r, n}\right)+c_{0, n}$, and by resorting to Schur's complement lemma to rewrite the quadratic objective (12a) in terms of linear matrix inequalities, one can obtain an SDP relaxation of (5) as [7]

$$
\min _{\mathbf{x} \succeq 0,\left\{\alpha_{n}\right\}} \sum_{n \in \mathcal{N}_{g}} \alpha_{n}
$$

subject to: (12b)-(12e); and

$$
\left[\begin{array}{cc}
\tilde{c}_{1, n} \operatorname{tr}\left\{\mathbf{X} \overline{\mathbf{Y}}_{n}\right\}+\tilde{c}_{0, n}-\alpha_{n} & \sqrt{\tilde{c}_{2, n}} \operatorname{tr}\left\{\mathbf{X} \overline{\mathbf{Y}}_{n}\right\} \\
\sqrt{\tilde{c}_{2, n}} \operatorname{tr}\left\{\mathbf{X} \overline{\mathbf{Y}}_{n}\right\} & -1
\end{array}\right] \preceq 0, n \in \mathcal{N}_{g}
$$

\section{ONLINE OPF With RENEWABLES}

The renewable generation is not dispatchable, and its availability can be predicted only with significant uncertainty. Since the power grid has to maintain the balance between power supply and demand at all times, integration of a high volume of renewables will markedly impact the stability of the grid.

One way to cope with the volatility of renewables is to rely on the spot market to buy or sell power to make up for the imbalance. Thus, a two-stage procedure is considered here, where the conventional generators are committed to produce power $P_{g, n}$ at each bus $n \in \mathcal{N}_{g}$ in the first stage, followed by real-time power transaction on the spot market in the second stage [23]. When the operator determines the amount of conventional generation in the first stage, it has to hedge against the uncertainties in the renewable generation. ${ }^{1}$ Since the OCO framework is suitable for this situation, we next brief the OCO approach.

\section{A. Online Convex Optimization}

The OCO model considers a repeated game between a player and an adversary [19]. (In the present setup, the utility that solves the OPF problem assumes the role of the player, while the renewable generation can be regarded as the adversary.) At time $t$, the player chooses an action $\mathbf{p}^{t} \in \mathcal{P}$, and subsequently the adversary reveals a convex function $c^{t}: \mathcal{P} \rightarrow \mathbb{R}$. As will be specified in the next subsection, $\mathbf{p}^{t}$ will be the vector of conventional generator outputs, and $c^{t}$ related to the OPF cost. Then, the player suffers the loss of amount $c^{t}\left(\mathbf{p}^{t}\right)$. The goal of the player is to minimize the so-called regret $R_{c}(T)$ over $T$ time slots, defined as

$$
R_{c}(T):=\sum_{t=1}^{T} c^{t}\left(\mathbf{p}^{t}\right)-\min _{\mathbf{p} \in \mathcal{P}} \sum_{t=1}^{T} c^{t}(\mathbf{p})
$$

which corresponds to the cost incurred by the player relative to the cost due to a single best action $\mathrm{p}^{*}:=$ $\arg \min _{\mathbf{p} \in \mathcal{P}} \sum_{t=1}^{T} c^{t}(\mathbf{p})$, which is selected with the benefit of knowing $c^{t}$ for all $t=1,2, \ldots, T$ in hindsight.

Under appropriate conditions, online iterative algorithms can be constructed, which yield $\mathbf{p}^{t}$ at time $t$, to achieve the regret upper-bounds that grow sublinearly in $T$; i.e., $R_{c}(T) / T \rightarrow 0$ as $T \rightarrow \infty$. This means that the online algorithms can eventually perform as well as the fixed action selected in hindsight in terms of the per-slot cost.

\section{B. Online OPF Formulation}

The online OPF problem can be stated as follows. At each time slot $t \in\{1,2, \ldots, T\}$, based on the realizations observed so far for the renewable generation, i.e., $\left\{P_{r, n}^{\tau}, Q_{r, n}^{\tau}\right\}_{\tau=1}^{t}$, find $\left\{P_{g, n}^{t+1}\right\}$ for $n \in \mathcal{N}_{g}$ to be used in the next time slot, so as to achieve regret $R_{c}(T)$ that is sublinear in $T$.

To address this problem without explicitly modeling the dynamics of renewable generation, the OCO approach is taken. The relevant cost function $c^{t}$ must capture the cost of conventional generation as well as the real-time energy transaction cost. Let $\left\{P_{s, n}\right\}$ and $\left\{Q_{s, n}\right\}$ be respectively the active and the reactive powers, purchased from the spot market through bus $n \in \mathcal{N}_{s}$, where $\mathcal{N}_{s}$ is the set of buses connected to the spot market. Select a convex function $g_{n}^{t}\left(P_{s, n}^{t}\right)$ to model the energy transaction cost at bus $n \in \mathcal{N}_{s}$ in time slot $t$. Upon

\footnotetext{
${ }^{1}$ The uncertainties in the load and the spot market prices can be accommodated similarly in our proposed approach.
} 
defining vector $\mathbf{p}_{g}^{t}:=\left\{P_{g, n}^{t}\right\}_{n \in \mathcal{N}_{g}}$, the overall cost $c^{t}$ in (14) to minimize the regret is

$$
c^{t}\left(\mathbf{p}_{g}^{t}\right):=\sum_{n \in \mathcal{N}_{g}} f_{n}\left(P_{g, n}^{t}\right)+g^{t}\left(\mathbf{p}_{g}^{t}\right)
$$

where

$$
g^{t}\left(\mathbf{p}_{g}^{t}\right):=\min _{\mathbf{X}^{t} \succeq \mathbf{0},\left\{P_{s, n}^{t}\right\},\left\{Q_{s, n}^{t}\right\},\left\{Q_{g, n}^{t}\right\}} \sum_{n \in \mathcal{N}_{s}} g_{n}^{t}\left(P_{s, n}^{t}\right)
$$

subject to:

$$
\begin{aligned}
& \underline{V}_{n}^{2} \leq X_{n n}^{t} \leq \bar{V}_{n}^{2}, \quad n \in \mathcal{N} \\
& X_{n n}^{t}+X_{n^{\prime} n^{\prime}}^{t}-X_{n n^{\prime}}^{t}-X_{n^{\prime} n}^{t} \leq \bar{V}_{n n^{\prime}}^{2}, \quad\left(n, n^{\prime}\right) \in \mathcal{E} \\
& \operatorname{tr}\left\{\mathbf{X}^{t} \overline{\mathbf{Y}}_{n}\right\}-P_{g, n}^{t}+P_{l, n}^{t}-P_{r, n}^{t}-P_{s, n}^{t}=0, n \in \mathcal{N} \\
& \operatorname{tr}\left\{\mathbf{X}^{t} \tilde{\mathbf{Y}}_{n}\right\}-Q_{g, n}^{t}+Q_{l, n}^{t}-Q_{r, n}^{t}-Q_{s, n}^{t}=0, n \in \mathcal{N} \\
& \underline{Q}_{g, n} \leq Q_{g, n}^{t} \leq \bar{Q}_{g, n}, \quad n \in \mathcal{N}_{g}
\end{aligned}
$$

where (16d)-(16e) are the balancing conditions with the energy to/from the spot market included; cf. (4).

Problem (16) is a static OPF problem with convex relaxation, aiming to minimize the spot market transaction cost, given the conventional generation $P_{g, n}^{t}$ fixed, and the renewable generation $P_{r, n}^{t}$ realized at time slot $t$. With strong duality holding for (16), $g^{t}\left(\mathbf{p}_{g}^{t}\right)$ is also convex [24]. Therefore, $c^{t}$ is convex as well.

\section{Online Mirror Descent-Based Algorithm}

A widely used algorithm for OCO problems is the online mirror descent (OMD) method [19]. Upon introducing the Bregman diverence $D_{\psi}\left(\mathbf{p}, \mathbf{p}^{\prime}\right)$ associated with a strongly convex function $\psi(\mathbf{p})$, as

$$
D_{\psi}\left(\mathbf{p}, \mathbf{p}^{\prime}\right):=\psi(\mathbf{p})-\psi\left(\mathbf{p}^{\prime}\right)-\left\langle\nabla \psi\left(\mathbf{p}^{\prime}\right), \mathbf{p}-\mathbf{p}^{\prime}\right\rangle
$$

where $\langle\cdot, \cdot\rangle$ denotes inner product, the OMD update is

$$
\mathbf{p}_{g}^{t+1}=\arg \min _{\mathbf{p}_{g} \in \mathcal{P}_{g}} \eta\left\langle\left(c^{t}\right)^{\prime}\left(\mathbf{p}_{g}^{t}\right), \mathbf{p}_{g}\right\rangle+D_{\psi}\left(\mathbf{p}_{g}, \mathbf{p}_{g}^{t}\right)
$$

where $\eta>0$ is a step size, $\left(c^{t}\right)^{\prime}\left(\mathbf{p}_{g}^{t}\right)$ is a subgradient of $c^{t}\left(\mathbf{p}_{g}\right)$ at $\mathbf{p}_{g}=\mathbf{p}_{g}^{t}$, and $\mathcal{P}_{g}$ is the feasible set for $\mathbf{p}_{g}^{t}$, representing the generator operational constraints such as $(5 \mathrm{c})-(5 \mathrm{~d})$. Note that a choice for $\left(c^{t}\right)^{\prime}\left(\mathbf{p}_{g}^{t}\right)$ is [cf. (15)]

$$
\left(c^{t}\right)^{\prime}\left(\mathbf{p}_{g}^{t}\right)=\nabla\left[\sum_{n \in \mathcal{N}_{g}} f_{n}\left(P_{g, n}^{t}\right)\right]-\boldsymbol{\lambda}_{g}^{t *}
$$

where $\boldsymbol{\lambda}_{g}^{t *}$ stands for the Lagrange multiplier vector associated with the balance constraints (16d) for $n \in \mathcal{N}_{g}$ [24].

For simplicity, we choose $\psi(\mathbf{p}):=\frac{1}{2}\|\mathbf{p}\|^{2}$. Then, $D_{\psi}\left(\mathbf{p}, \mathbf{p}^{\prime}\right)=\frac{1}{2}\left\|\mathbf{p}-\mathbf{p}^{\prime}\right\|^{2}$, and (18) becomes

$$
\mathbf{p}_{g}^{t+1}=\arg \min _{\mathbf{p}_{g} \in \mathcal{P}_{g}} \frac{1}{2}\left\|\mathbf{p}_{g}-\mathbf{p}_{g}^{t}+\eta\left(c^{t}\right)^{\prime}\left(\mathbf{p}_{g}^{t}\right)\right\|^{2}
$$

yielding a projected subgradient method [25]. Under mild regularity conditions, the OMD update can be shown to achieve a regret on the order of $O(\sqrt{T})$ [19].

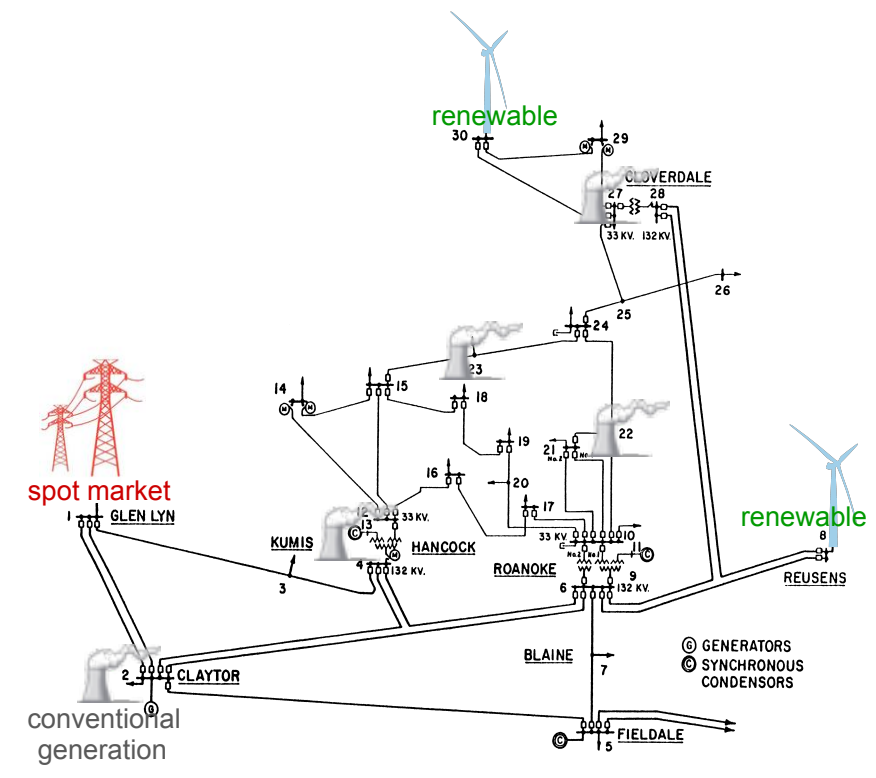

Fig. 1. A 30-bus test case

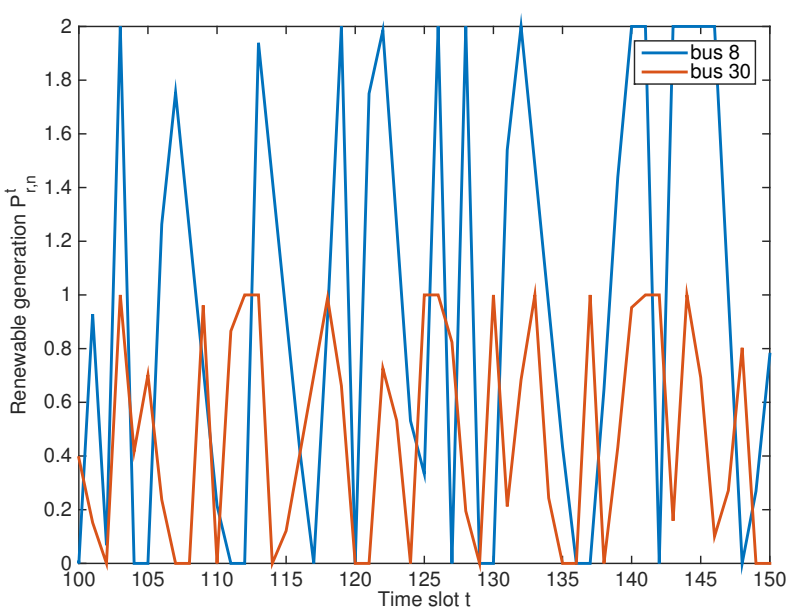

Fig. 2. Simulated renewable generation

\section{Numerical Tests}

To evaluate the proposed algorithm, simulated tests were performed using a 30-bus IEEE test case adapted from [26], whose topology is depicted in Fig. 1. Also shown in the figure are one bus $\mathcal{N}_{s}=\{1\}$ connected to the spot market, two buses $\mathcal{N}_{r}=\{8,30\}$ with renewable generation, and five buses $\mathcal{N}_{g}=\{2,13,22,23,27\}$ with conventional generation. Quadratic functions were employed for the costs of conventional generation $\left\{f_{n}(\cdot)\right\}_{n \in \mathcal{N}_{g}}$ as well as for the spot market cost $g_{1}^{t}(\cdot)$. For simplicity, the loads and the spot market prices are held fixed over time in the tests.

To facilitate the simulation of renewable generation with variable degrees of volatility, the model for wind turbine generation used in [20] was adopted. In this model, the power production ramps from 0 to the maximum capacity in $T_{u p}$ time 


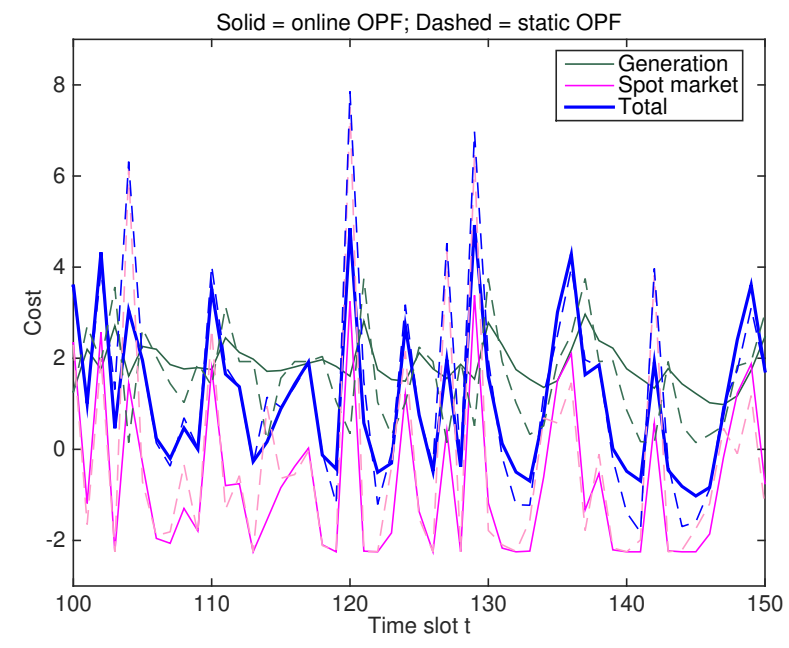

Fig. 3. Cost of online and static OPF

slots, stays at the maximum for $T_{o n}$ slots, ramps down to 0 in $T_{\text {down }}$ slots, and stays at 0 for $T_{o f f}$ slots, where $T_{u p}, T_{o n}$, $T_{\text {down }}$ and $T_{\text {off }}$ are sampled from exponential distribution with mean $\mu_{T}$. A realization of the outputs from two wind turbine generators with $\mu_{T}=1$ is shown in Fig. 2 .

In addition to the proposed online OPF algorithm, a benchmark static OPF algorithm was run for comparison. In the static OPF, at the end of time slot $(t-1),\left\{P_{g, n}^{t}\right\}$ is determined by solving an OPF problem assuming that the production of renewable generation in slot $t$ will be the same as in slot $(t-1)$ (persistence forecast). Specifically, one solves

$$
\min _{\substack{\mathbf{X}^{t} \succeq \mathbf{0},\left\{P_{g, n}^{t}\right\},\left\{Q_{g, n}^{t}\right\} \\\left\{P_{s, n}^{t}\right\},\left\{Q_{s, n}^{t}\right\}}} \sum_{n \in \mathcal{N}_{g}} f_{n}\left(P_{g, n}^{t}\right)+\sum_{n \in \mathcal{N}_{s}} g_{n}^{t-1}\left(P_{s, n}^{t}\right)
$$

subject to: (16b)-(16c); and

$$
\begin{aligned}
& \underline{P}_{g, n} \leq P_{g, n}^{t} \leq \bar{P}_{g, n}, \quad n \in \mathcal{N}_{g} \\
& \underline{Q}_{g, n} \leq Q_{g, n}^{t} \leq \bar{Q}_{g, n}, \quad n \in \mathcal{N}_{g} \\
& \operatorname{tr}\left\{\mathbf{X}^{t} \overline{\mathbf{Y}}_{n}\right\}-P_{g, n}^{t}+P_{l, n}^{t-1}-P_{r, n}^{t-1}-P_{s, n}^{t}=0, n \in \mathcal{N} \\
& \operatorname{tr}\left\{\mathbf{X}^{t} \tilde{\mathbf{Y}}_{n}\right\}-Q_{g, n}^{t}+Q_{l, n}^{t-1}-Q_{r, n}^{t-1}-Q_{s, n}^{t}=0, n \in \mathcal{N}
\end{aligned}
$$

In time slot $t$, after the actual realization of the renewable generation is revealed, OPF (16) is solved again to determine the spot market transaction. The total cost of the static OPF is given by $\sum_{n \in \mathcal{N}_{g}} f_{n}\left(P_{g, n}^{t}\right)+g^{t}\left(\mathbf{p}_{g}^{t}\right)$. Note that this scheme requires solving two OPF problems per time slot, while the proposed online OPF solves OPF only once per time slot.

Fig. 3 shows the evolution of the total cost (in addition to the costs for conventional generation and spot market transaction components) for the online and static OPF schemes when the renewable generation is as given in Fig. 2. The value of $\eta=0.05$ was used for online OPF. It can be seen that online OPF achieves a cost lower than its static counterpart when there is a sudden drop in the renewable

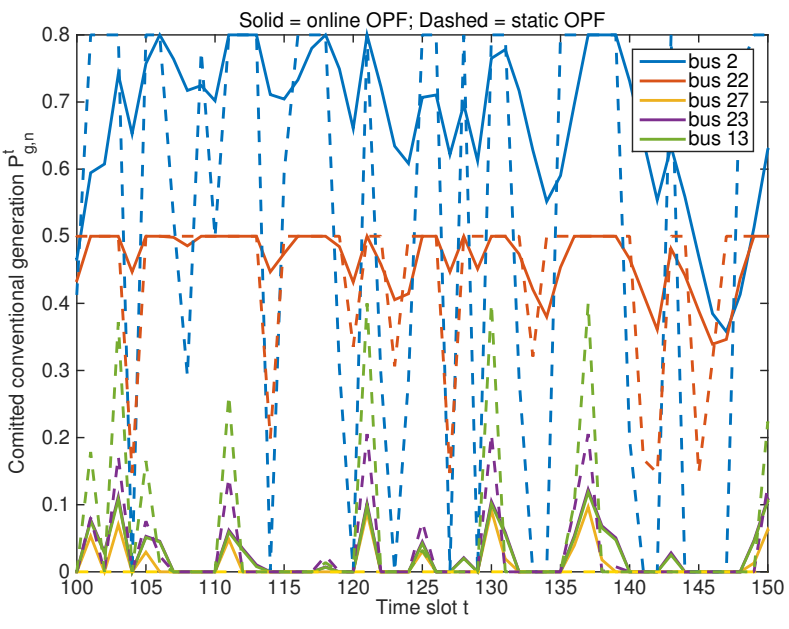

Fig. 4. Conventional generation

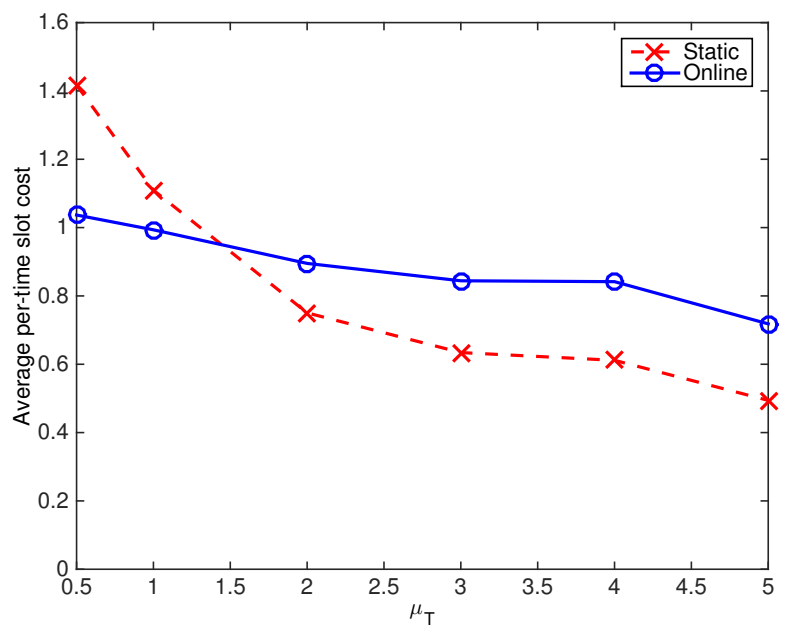

Fig. 5. Average cost for online and static OPF

generation. This is because the static OPF fully trusts the forecast for renewable generation and decreases the committed conventional generation significantly. Thus, when there is a big drop in the renewable generation, the spot market cost soars. Such a pattern is corroborated in Fig. 4, which depicts the committed conventional generation at each time slot for online and static OPF schemes.

Fig. 5 shows the average cost for online and static alternatives when $\mu_{T}$ is varied. Recall that small $\mu_{T}$ yields high volatility in the renewable generation. The figure shows that the online OPF is advantageous when the renewable generation fluctuates considerably. The static OPF is superior when $\mu_{T}>1.5$. However, it is emphasized here that static OPF solves two OPF problems per slot, whereas online OPF solves only one; and that the learning parameter $\eta$ was not fully optimized in these test results. 


\section{CONClusion}

The OPF problem for power systems with renewable generation was tackled. To cope with pronounced variability in the renewable generation output, a hedging strategy was derived using the OCO framework. The proposed online algorithm updates the level of conventional generation for the next time slot based on the shadow prices of the convex relaxation of an OPF problem determining optimal spot market transactions in the current time slot. Compared to a static alternative, which solves one extra OPF based on the forecasted renewables output, the proposed online scheme achieves lower average cost under high volatility of renewables. In our future research, tests based on real data will be performed, in addition to rigorous regret analysis under ramping constraints.

\section{REFERENCES}

[1] J. Carpentier, "Contribution to the economic dispatch problem," Bull. Soc. Franc. Elect., vol. 8, no. 3, pp. 431-447, 1962.

[2] A. J. Wood and B. F. Wollenberg, Power Generation, Operation and Control, 3rd ed. Hoboken, NJ: John Wiley \& Sons, 2013.

[3] J. A. Momoh, M. E. El-Hawary, and R. Adapa, "A review of selected optimal power flow literature to 1993. Part I: Nonlinear and quadratic programming approaches," IEEE Trans. Power Syst., vol. 14, no. 1, pp. 96-104, Feb. 1999.

[4] _ - "A review of selected optimal power flow literature to 1993. Part II: Newton, linear programming and interior point methods," IEEE Trans. Power Syst., vol. 14, no. 1, pp. 104-111, Feb. 1999.

[5] Z. Qiu, G. Deconinck, and R. Belmans, "A literature survey of optimal power flow problems in the electricity market context," in Proc. of the Power Syst. Conf. Expo., Seattle, WA, Mar. 2009, pp. 1-6.

[6] X. Bai, H. Wei, K. Fujisawa, and Y. Wang, "Semidefinite programming for optimal power flow problems," Int'l. J. Electr. Power \& Energy Syst., vol. 30, no. 6-7, pp. 383-392, Jul.-Sept. 2008.

[7] J. Lavaei and S. H. Low, "Zero duality gap in optimal power flow problem," IEEE Trans. Power Syst., vol. 27, no. 1, pp. 92-107, Feb. 2012.

[8] S. H. Low, "Convex relaxation of optimal power flow-Part I: Formulations and equivalence," IEEE Trans. Contr. of Net. Syst., vol. 1, no. 1, pp. 15-27, Mar. 2014.

[9] - "Convex relaxation of optimal power flow-Part II: Exactness," IEEE Trans. Contr. of Net. Syst., vol. 1, no. 2, pp. 117-189, May 2014

[10] B. C. Lesieutre, D. K. Molzhan, A. R. Borden, and C. L. DeMarco, "Examining the limits of the application of semidefinite programming to power flow problems," in Proc. of the 49th Annual Allerton Conf. Commun. Control Comput., Monticello, IL, Sep. 2011, pp. 1492-1499.

[11] D. K. Molzhan, J. T. Holzer, B. C. Lesieutre, and C. L. DeMarco, "Implementation of a large-scale optimal power flow solver based on semidefinite programming," IEEE Trans. Power Syst., vol. 28, no. 4, pp. 3987-3998, Nov. 2013.
[12] D. Bienstock, M. Chertkov, and S. Harnett, "Chance constrained optimal power flow: Risk-aware network control under uncertainty," Feb. 2013. [Online]. Available: http://arxiv.org/abs/1209.5779

[13] Y. Zhang, N. Gatsis, and G. B. Giannakis, "Robust energy management for microgrids with high-penetration renewables," IEEE Trans. Sustain. Energy, vol. 4, no. 4, pp. 944-953, Oct. 2013.

[14] J. Liang, G. K. Venayagamoorthy, and R. G. Harley, "Wide-area measurement based dynamic stochastic optimal power flow control for smart grids with high variability and uncertainty," IEEE Trans. Smart Grid, vol. 3, no. 1, pp. 59-69, Mar. 2012.

[15] M. D. Ilić, L. Xie, and J.-Y. Joo, "Efficient coordination of wind power and price-responsive demand-Part I: Theoretical foundations," IEEE Trans. Power Syst., vol. 26, no. 4, pp. 1875-1884, Nov. 2011.

[16] G. Valverde and T. V. Cutsem, "Model predictive control of voltages in active distribution networks," IEEE Trans. Smart Grid, vol. 4, no. 4, pp. 2152-2161, Dec. 2013

[17] M. J. Dolan, E. M. Davidson, I. Kockar, G. W. Ault, and S. D. J. McArthur, "Distribution power flow management utilizing an online optimal power flow technique," IEEE Trans. Power Syst., vol. 27, no. 2, pp. 790-799, May 2012.

[18] V. K. Garg, T. S. Jayram, and B. Narayanaswamy, "Online optimization with dynamic temporal uncertainty: Incorporating short term predictions for renewable integration in intelligent energy systems," in Proc. of the 27th AAAI Conf. Artificial Intell., Bellevue, WA, Jul. 2013, pp. 12911297.

[19] S. Shalev-Shwartz, "Online learning and online convex optimization," Foundations and Trends in Machine Learning, vol. 4, no. 2, pp. 107194, Mar. 2012.

[20] B. Narayanaswamy, V. K. Garg, and T. S. Jayram, "Online optimization for the smart (micro) grid," in Proc. of the 3rd Intl. Conf. Future Energy Syst. (e-Energy '12), Madrid, Spain, May 2012, pp. 1-10.

[21] S.-J. Kim, G. Wang, and G. B. Giannakis, "Online semidefinite programming for power system state estimation," in Proc. of the IEEE Intl. Conf. Acoustics Speech Sig. Proc. (ICASSP), Florence, Italy, May 2014, pp. 6024-6027.

[22] S.-J. Kim and G. B. Giannakis, "Real-time electricity pricing for demand response using online convex optimization," in Proc. of the IEEE PES Innovative Smart Grid Technologies Conf. (ISGT), Washington, DC, Feb. 2014, pp. 1-5.

[23] D. Phan and S. Ghosh, "Two-stage stochastic optimization for optimal power flow under renewable generation uncertainty," ACM Trans. Modeling Comput. Simul., vol. 24, no. 1, pp. 2:1-2:24, Jan. 2014.

[24] S. Boyd and L. Vandenberghe, Convex Optimization. New York, NY: Cambridge University Press, 2004.

[25] A. Beck and M. Teboulle, "Mirror descent and nonlinear projected subgradient methods for convex optimization," Oper. Res. Lett., vol. 31, no. 3, pp. 167-175, 2003.

[26] R. D. Zimmerman, C. E. Murillo-Sánchez, and R. J. Thomas, "MATPOWER: Steady-state operations, planning and analysis tools for power systems research and education," IEEE Trans. Power Syst., vol. 26, no. 1, Feb. 2011. 